\title{
Robust estimations of current velocities with four-beam
}

\section{broadband ADCPs}

M. Gilcoto ${ }^{1}$, Emlyn Jones ${ }^{2}$, Luis Fariña-Busto ${ }^{3}$

${ }^{1}$ Departamento de Oceanoloxía, Instituto de Invetigacións Mariñas (IIM), Consejo

Superior de Investigaciones Científicas (CSIC), Vigo, Spain

${ }^{2}$ School of Chemistry, Physics and Earth Sciences, Flinders University of South Australia, Australia

${ }^{3}$ Física de la Tierra, Astronomía y Astrofísica, Universidad de Vigo, Universidad de Vigo, Vigo, Spain

Version 4: June $1^{\text {st }}, 2009$. 



\section{Abstract}

An extended explanation of the hypothesis and equations traditionally used to transform between four-beam ADCP radial beam velocities and current velocity components is presented. This explanation includes a dissertation about the meaning of the RD Instrument error velocity and the description of the standard beam to current components transformation as a least squares solution. Afterwards, the variancecovariance matrix associated with the least squares solution is found. Then, a robust solution for transforming radial beam velocities into current components is derived under the formality of a weighted least squares approach. The associated variancecovariance matrix is also formulated and theoretically proves that the modulus of its elements will be generally lower than the corresponding modulus of the variancecovariance matrix associated with the standard least squares solution. Finally, we present a comparison between the results obtained using the standard least squares solution, with the results of the weighted least squares method, using a high-resolution ADCP dataset. The results show that, in this case, the weighted least squares solution provides variance estimations that are $4 \%$ lower over the entire record period (8 days) and 7\% lower during a shorter more energetic period (12 hours). 


\section{1. Introduction}

The observation of current velocities in the ocean and coastal seas has been greatly improved with the aid of Acoustic Doppler Current Profilers (ADCP). The ADCP is a sonar (Acoustic) instrument that uses sound to sense current velocities at set distances away from its transducer head, creating a profile of the currents throughout the water column.

There are several companies manufacturing ADCPs, producing several models and types of ADCPs: self contained ADCP, vessel mounted ADCP, horizontal ADCPs, ADCPs with three, four or five transducers, ADCPs are even available with phase arrayed transducers and with different working frequencies. Depending on the application, some ADCPs are capable of using different ways of transmitting and processing the sound signals (pulse-to-pulse incoherent, pulse-to-pulse coherent and broadband). They have their own devoted literature the scope of which ranges from technical papers regarding the internal signal processing methods and performance of ADCPs (Chereskin and Harding, 1993; Lhermitte and Serafin, 1984) to papers about mooring vibration induced errors (Hamilton et al., 1997), the difficult task of processing vessel mounted ADCP data (New, 1992) and the errors introduced by the vertical diel migration of zooplankton (Ott, 2005). Furthermore, ADCPs can be used for turbulence estimation (Gargett, 1994, Stacey et al., 1999), observations of sea surface conditions (van Haren, 2001; Visbeck and Fischer, 1995) and many more field and laboratory applications.

However, and despite all the articles focusing on ADCP data processing techniques already cited, it has not been established a procedure to estimate the velocity error of the velocity components of the currents yet. Beyond the obvious benefits of having velocity current measurements accompanied with their corresponding error 
1 estimates there are several ADCP applications that certainly would improve their

2 performance. First, the errors would help in the screening of the ADCP recorded data, it

3 is easy and intuitive to reject values with higher error than a certain a priori established

4 limit. Secondly, the inverse methods used to process LADCP (Lowered ADCP) data

5 (Visbeck, 2002) and the calculus of Reynolds stresses with ADCPs (Stacey et al., 1999;

6 Williams and Simpson, 2004) will take advantage of the error estimations.

7 We will center the present work on one of the commonest ADCPs, the four

8 beam broadband unit (four transducers with broadband signal processing techniques),

9 and in the transformation of its radial beam velocities into current velocity components.

10 Since the current velocity vector is composed by three orthogonal components the

11 existence of four or more transducers oriented in different directions will provide

12 additional estimates for the velocity components. On the other hand, the broadband

13 signal processing techniques allows the independent estimation, for each beam and cell

14 in the profile, of the radial velocities variances generated by the Doppler noise. These

15 two characteristics of the broadband four-beams ADCPs can be combined to transform

16 the radial beam velocities into current velocity components through weighted least

17 squares (WLS) methods, which will generate robust estimates of the velocity currents

18 and estimates of their associated errors. Thus, the aim of this article is to show a new

19 processing technique of raw Broadband ADCP data, which generates more robust

20 velocity estimates than the standard processing technique and allows the estimation of

21 the corresponding velocity errors.

\section{Posing the problem}

An ADCP needs one transducer for every velocity component we want to

25 measure and, obviously, each transducer must be oriented in a different direction. 
1 Usually, the ADCPs have four transducers distributed in two pairs, each pair drawing a

2 Janus configuration i.e. one transducer situated in front of the other and looking in

3 opposite directions (Figure 1). So a four-beam ADCP has an extra radial beam velocity

4 measurement than needed. The ADCPs divides each beam into vertical segments (bins

5 or cells) and measures the average radial beam velocities of the scattering particles

6 included in the volume enclosed by the beam in each bin. This results in an estimate of

7 velocity profile throughout water column for each beam. Since the transducers are

8 spread in different directions the bins of each beam are not located in the same

9 geographical position, meaning the transducers are not sensing the same volume of

10 water. For a particular bin, the radial beam velocity $\left(\vec{b}_{i}\right)$ for each transducer $(i=1-4)$

11 generated by the water flow in each beam $\left(\vec{V}_{i}\right)$ may be expressed mathematically as

$$
\begin{aligned}
& b_{1}=V_{1, X} \cdot \sin \beta+V_{1, Z} \cdot \cos \beta \\
& b_{2}=-V_{2, X} \cdot \sin \beta+V_{2, Z} \cdot \cos \beta \\
& b_{3}=-V_{3, Y} \cdot \sin \beta+V_{3, Z} \cdot \cos \beta \\
& b_{4}=V_{4, Y} \cdot \sin \beta+V_{4, Z} \cdot \cos \beta
\end{aligned}
$$

13 where $V_{i, X}, V_{i, Y}$ and $V_{i, Z}$ are the $\mathrm{X}, \mathrm{Y}$ and $\mathrm{Z}$ velocity components of the current in each

14 transducer beam (i) and $\beta$ is the slant angle of the ADCP transducer ceramics (Fig. 1).

15 The former velocity components of the current were expressed in the internal coordinate system of the ADCP (system XYZ) as depicted in Figure 1. The equations (1) are easily deduced from the trigonometric relations shown in Figure 1.

With 8 velocity components of currents as unknowns but only with 4 different radial beam velocities as known information, the system of equations (1) is underdetermined. The best way to fix this issue is to recall the so called homogeneous velocity field hypothesis, i.e. to assume that the currents are homogeneous in the region

22 illuminated by the four beams, meaning 


$$
V_{X}=V_{i, X}, V_{Y}=V_{i, Y} \text { and } V_{Z}=V_{i, Z}(i=1-4)
$$

$$
\overrightarrow{\mathbf{b}}=\left(\begin{array}{ccc}
\sin \beta & 0 & \cos \beta \\
-\sin \beta & 0 & \cos \beta \\
0 & -\sin \beta & \cos \beta \\
0 & \sin \beta & \cos \beta
\end{array}\right) \cdot\left(\begin{array}{c}
V_{X} \\
V_{Y} \\
V_{Z}
\end{array}\right)=\mathbf{A} \cdot \overrightarrow{\mathbf{V}}_{X Y Z}
$$

and in East/North/Up (ENU) velocities (see Appendix A)

$$
\overrightarrow{\mathbf{b}}=\mathbf{A} \cdot \overrightarrow{\mathbf{V}}_{X Y Z}=\mathbf{A} \cdot \mathbf{M} \cdot\left(\begin{array}{c}
u \\
v \\
w
\end{array}\right)=\mathbf{T} \cdot \overrightarrow{\mathbf{V}}_{E N U}
$$

5 defining $\mathbf{T}=\mathbf{A} \cdot \mathbf{M}$, so the new ENU to beam transformation matrix $\mathbf{T}$ is also function of

6 the attitude angles ( $\theta$ the heading, $\phi$ the pitch, and $\varphi$ the roll, $\mathbf{T}=\mathbf{T}(\theta, \phi, \varphi)$ ). Thus, after

7 calling the assumption (2) we obtain an overdetermined system of equations (eq. (3) or

8 eq. (4) for XYZ or ENU velocities, respectively) with 3 unknowns and 4 equations.

At this point, in what we are interested is in seeking the best way to solve the system of equations (3) regardless the homogeneous velocity field assumption.

11 Therefore, first we will refresh the standard solution given to (3) and then, in the

12 following sections, least squares techniques will be applied to find optimum solutions.

\section{Standard solution}

Following Lohrmann et al. (1990), and applying the homogeneous flow assumption by transducer pairs $\left(V_{12, X}=V_{1, X}=V_{2, X}, V_{12, Z}=V_{1, Z}=V_{2, Z}, V_{34, Y}=V_{3, Y}=V_{4, Y}\right.$ and $V_{34, Z}=V_{3, Z}=V_{4, Z}$ ) one may derive from eqs. (1), the following set of equations:

$$
V_{12, X}=\frac{1}{2 \cdot \sin \beta} \cdot\left(b_{1}-b_{2}\right) \text {, }
$$

$$
V_{12, Z}=\frac{1}{2 \cdot \cos \beta} \cdot\left(b_{1}+b_{2}\right),
$$




$$
\begin{aligned}
& V_{34, Y}=\frac{1}{2 \cdot \sin \beta} \cdot\left(b_{4}-b_{3}\right), \\
& V_{34, Z}=\frac{1}{2 \cdot \cos \beta} \cdot\left(b_{3}+b_{4}\right) .
\end{aligned}
$$

3 Giving one estimate for each component of the horizontal velocity $\left(V_{12, X}\right.$ and $\left.V_{34, Y}\right)$ and

4 two estimates of the vertical component of the velocity field $\left(V_{12, Z}\right.$ and $\left.V_{34, Z}\right)$. The next

5 best step is to average the vertical components to obtain a more robust estimation

$$
V_{Z}=-\frac{1}{4 \cdot \cos \beta} \cdot\left(b_{1}+b_{2}+b_{3}+b_{4}\right)
$$

7 and calculate an "error velocity” with their difference

$$
e_{V_{Z}}=V_{12, Z}-V_{34, Z}=\frac{1}{2 \cdot \cos \beta} \cdot\left(b_{1}+b_{2}-b_{3}-b_{4}\right)
$$

9 The so called error velocity may be a good proxy to evaluate the validity of the homogeneous velocity field assumption ( $\mathrm{Lu}$ and Lueck, 1999a), but it is not a

11 quantitative measure of the error of the velocity current. A high value will indicate a

12 deviation from homogeneous flow, whereas a low error velocity might confirm it (at

13 least will confirm the existence of an homogeneous vertical velocity field when $\phi \approx 0$ and

$14 \varphi \approx 0$ ) and justify the set of equations (5), (7) and (9) as the sought solution for (3). This

15 set of equations configures the standard solution and matches the one shown by one of

16 the most renowned ADCP's manufactures, RD Instruments (RDI), in its manual of

17 formulas and calculations for a convex upward looking ADCP (R. D. Instruments, 18 1997).

A tacit extension of the homogeneous flow field supposition from pairs of beams

20 to the whole set of beams has been made when including eq. (9) in the standard

21 solution. In any case, the extension is mandatory if the objective is to reconstruct the three dimensional (3D) velocity field or we would have just a horizontal component of 
1 the velocity (namely, $V_{12, X}$ ) in the region illuminated by one pair, the other horizontal

2 component $\left(V_{34, Y}\right)$ in the region of the other pair and two vertical components $\left(V_{12, Z}\right.$ and

$3 V_{34, Z}$ ), one for each beam pair.

4

It is worth noting that the RDI Company does not use eq. (10) to calculate what we have called the RDI error velocity $\left(e_{R D I}\right)$ although in page 14 of Gordon (1996) they define it as "the difference between the two estimates of vertical velocity". The formula used by the RDI's ADCPs to calculate $e_{R D I}$ is (page 10 in R. D. Instruments (1997))

$$
e_{R D I}=\frac{1}{\sqrt{2} \cdot 2 \cdot \sin \beta} \cdot\left(b_{1}+b_{2}-b_{3}-b_{4}\right)
$$

There is a factor (eq. (12)) between the RDI and the standard velocity error (eq. (10)) that makes the former quotation misleading. In fact, some researchers have found unclear what the RDI error velocity actually measures (Ott, 2005). Put it simple, it is not a real error estimate of the current velocity, it is a kind of proxy.

$$
e_{R D I}=\frac{2 \cdot \cos \beta}{\sqrt{2} \cdot 2 \cdot \sin \beta} \cdot e_{V_{Z}}
$$

\section{Four-beam standard least squares solution}

The derivation of the standard solution, carried out in the latter section, made clear that it is compulsory to recall the homogeneity flow assumption in order to estimate the $3 \mathrm{D}$ velocity vector from the measurements taken with a four beam ADCP. Hence, in the following we will pragmatically accept it as part of the solution. Then we will drop the beam/transducers subscripts in the current velocity components, although we will retain them in the radial beam velocities (BEAM coordinates).

It should be possible to get more information from the ADCP and maybe improve in some (statistical) degree the transformation from BEAM to XYZ velocities. Let us begin with the system of equations (3) that transform the XYZ velocity field into 
1 radial beam velocities, under the assumption of horizontal homogeneity and in the

2 presence of noise ( $\overrightarrow{\mathbf{e}})$

3

$$
\begin{aligned}
& \left(\begin{array}{l}
b_{1} \\
b_{2} \\
b_{3} \\
b_{4}
\end{array}\right)=\left(\begin{array}{ccc}
\sin \beta & 0 & \cos \beta \\
-\sin \beta & 0 & \cos \beta \\
0 & -\sin \beta & \cos \beta \\
0 & \sin \beta & \cos \beta
\end{array}\right) \cdot\left(\begin{array}{l}
V_{X} \\
V_{Y} \\
V_{Z}
\end{array}\right)+\left(\begin{array}{l}
e_{1} \\
e_{2} \\
e_{3} \\
e_{4}
\end{array}\right) \\
& \overrightarrow{\mathbf{b}}=\mathbf{A} \cdot \overrightarrow{\mathbf{V}}_{X Y Z}+\overrightarrow{\mathbf{e}}
\end{aligned}
$$

4 We can seek a least squares solution to the system (13), following the matrix methods

5 described in chapter 3 of Wunsch (1996). The optimal estimation $\left(\hat{\mathbf{V}}_{X Y Z}\right)$ for $\overrightarrow{\mathbf{V}}_{X Y Z}$ is 6 then given by the so called normal equations:

7

8

$$
\begin{gathered}
\hat{\mathbf{V}}_{X Y Z}=\left(\mathbf{A}^{T} \cdot \mathbf{A}\right)^{-1} \cdot \mathbf{A}^{T} \cdot \overrightarrow{\mathbf{b}} \\
\left(\begin{array}{l}
\hat{V}_{X} \\
\hat{V}_{Y} \\
\hat{V}_{Z}
\end{array}\right)=\left(\begin{array}{cccc}
\frac{1}{2 \cdot \sin \beta} & -\frac{1}{2 \cdot \sin \beta} & 0 & 0 \\
0 & 0 & -\frac{1}{2 \cdot \sin \beta} & \frac{1}{2 \cdot \sin \beta} \\
\frac{1}{4 \cdot \cos \beta} & \frac{1}{4 \cdot \cos \beta} & \frac{1}{4 \cdot \cos \beta} & \frac{1}{4 \cdot \cos \beta}
\end{array}\right) \cdot\left(\begin{array}{l}
b_{1} \\
b_{2} \\
b_{3} \\
b_{4}
\end{array}\right)
\end{gathered}
$$

10 i.e. we recover exactly the standard solution for the velocity components (eqs. (5), (7)

11 and (9)), thus it will be called standard least squares (SLS) solution. On the other hand,

12 if we consider that only the instrumental noise is working against our velocity optimal

13 estimation (eq. (15)) and if we know the variance-covariance matrix $\left(\mathbf{C}_{\mathbf{e e}}\right)$ of the beam

14 radial velocities

$$
\mathbf{C}_{\mathbf{e e}}=\left(\begin{array}{cccc}
\sigma_{b_{1}}^{2} & 0 & 0 & 0 \\
0 & \sigma_{b_{2}}^{2} & 0 & 0 \\
0 & 0 & \sigma_{b_{3}}^{2} & 0 \\
0 & 0 & 0 & \sigma_{b_{4}}^{2}
\end{array}\right)
$$


1 (only instrumental noise, so the covariances are null) we will be able to obtain the

2 variance-covariance matrix $\left(\mathbf{C}_{\hat{\mathbf{V}}_{X Y Z}}\right)$ of the estimation $\left(\hat{\mathbf{V}}_{X Y Z}\right)$

$$
\mathbf{C}_{\hat{\mathbf{V}} \hat{\mathbf{V}}_{X Y Z}}=\left(\begin{array}{ccc}
\sigma_{\hat{V}_{X}}^{2} & \sigma_{\hat{V}_{X} \hat{V}_{Y}} & \sigma_{\hat{V}_{X} \hat{V}_{Z}} \\
\sigma_{\hat{V}_{Y} \hat{V}_{X}} & \sigma_{\hat{V}_{Y}}^{2} & \sigma_{\hat{V}_{Y} \hat{V}_{Z}} \\
\sigma_{\hat{V}_{Z} \hat{V}_{X}} & \sigma_{\hat{V}_{Z} \hat{V}_{Y}} & \sigma_{\hat{V}_{Z}}^{2}
\end{array}\right)=\left(\mathbf{A}^{T} \cdot \mathbf{A}\right)^{-1} \cdot \mathbf{A}^{T} \cdot \mathbf{C}_{\mathbf{e e}} \cdot \mathbf{A} \cdot\left(\mathbf{A}^{T} \cdot \mathbf{A}\right)^{-1}
$$

4

$$
\mathbf{C}_{\hat{\mathbf{v}} \hat{\mathbf{v}}_{X Y Z}}=\left(\begin{array}{ccc}
\frac{\sigma_{b_{1}}^{2}+\sigma_{b_{2}}^{2}}{4 \cdot \sin ^{2} \beta} & 0 & \frac{\sigma_{b_{1}}^{2}-\sigma_{b_{2}}^{2}}{8 \cdot \sin \beta \cdot \cos \beta} \\
0 & \frac{\sigma_{b_{3}}^{2}+\sigma_{b_{4}}^{2}}{4 \cdot \sin ^{2} \beta} & \frac{\sigma_{b_{4}}^{2}-\sigma_{b_{3}}^{2}}{8 \cdot \sin \beta \cdot \cos \beta} \\
\frac{\sigma_{b_{1}}^{2}-\sigma_{b_{2}}^{2}}{8 \cdot \sin \beta \cdot \cos \beta} & \frac{\sigma_{b_{4}}^{2}-\sigma_{b_{3}}^{2}}{8 \cdot \sin \beta \cdot \cos \beta} & \frac{\sigma_{b_{1}}^{2}+\sigma_{b_{2}}^{2}+\sigma_{b_{3}}^{2}+\sigma_{b_{4}}^{2}}{16 \cdot \cos ^{2} \beta}
\end{array}\right)
$$

5 where, for a broadband ADCP, the variance of the radial velocities due to instrumental

6 noise can be estimated with (Brumley et al., 1991; Pinkel and Smith, 1992; R. D.

7 Instruments, 1996):

$$
\sigma_{b_{i, j}}^{2}=\left(\frac{1.5 \cdot V_{a}}{2 \cdot \pi}\right)^{2} \cdot \frac{C y c \cdot C \cdot \cos \beta}{F_{0} \cdot D} \cdot\left(\frac{1}{R_{i, j}^{2}}-1\right)
$$

9 In the last equation $R_{i, j}$ is the correlation of the beam $i$ in the cell $j$ where the calculations

10 are being done (it is an output of the RDI ADCP), $D$ is the depth cell length (m), Cyc is

11 the number of carrier cycles per pulse code element (4 or 16 when the ADCP is setup

12 with WB0 or WB1 commands, respectively), $C$ is the speed of the sound (m/s), $F_{0}$ is the

13 working frequency of the $\mathrm{ADCP}(\mathrm{Hz})$ and $V_{a}$ is the ambiguity velocity calculated as

$$
V_{a}=\frac{C^{2} \cdot \cos \beta}{8 \cdot F_{0} \cdot L_{R D I}}
$$

15 Following the Appendix A of Hummon and Firing (2003), where $L_{R D I}$ is the lag (in m/s

16 to obtain $V_{a}$ in the same units) scaled by RDI from time to vertical distance and stored

17 for each measured profile in the RDI raw data files. 
The analytical expressions in East/North/Up (ENU) coordinates corresponding

2 to the SLS solution for eqs. (4) are quite complicated, though they can be inferred from

3 the ENU to XYZ matrices $\mathbf{M}$ and $\mathbf{N}$ presented in Appendix A. In order to simplify the

4 notation, and taking advantage of the analytical formulas already shown in the

5 paragraphs above, the SLS ENU solutions in matrix form are:

6

7

8

$19 \quad\left(\begin{array}{c}\tilde{V}_{X} \\ \tilde{V}_{Y} \\ \tilde{V}_{Z}\end{array}\right)=\left(\mathbf{A}^{T} \cdot \mathbf{C}_{\mathbf{e e}}^{-1} \cdot \mathbf{A}\right)^{-1} \cdot \mathbf{A}^{T} \cdot \mathbf{C}_{\mathbf{e e}}^{-1} \cdot \overrightarrow{\mathbf{b}}$ covariance matrix of the solution. as the general-case optimum solution:

with $\hat{\mathbf{V}}_{X Y Z}$ and $\mathbf{C}_{\hat{\mathbf{V}} \hat{\mathbf{V}}_{X Y Z}}$ given by (15) and (18), respectively.

Hence, applying the least squares optimum solution one may recover exactly the standard solution (section 3) plus an estimation of the error based on the variance-

\section{Weighted least squares solution}

In the case of a malfunctioning transducer, one will obtain huge errors when using the SLS solution (eqs. (15) and (18)). In order to manage the errors, solve threebeam problems without loosing error estimates and improve the robustness of the fourbeam velocity estimations we propose a weighted least square (WLS) solution (eq. (23))

20 and its associated variance-covariance matrix (eq. (24)) due to instrumental noise as

21 error estimate: 


$$
\mathbf{C}_{\tilde{\mathbf{V}} \tilde{V}_{X Y Z}}=\left(\begin{array}{ccc}
\sigma_{\tilde{V}_{X}}^{2} & \sigma_{\tilde{V}_{X} \tilde{V}_{Y}} & \sigma_{\tilde{V}_{X} \tilde{V}_{Z}} \\
\sigma_{\tilde{V}_{Y} \tilde{V}_{X}} & \sigma_{\tilde{V}_{Y}}^{2} & \sigma_{\tilde{V}_{Y} \tilde{V}_{Z}} \\
\sigma_{\tilde{V}_{Z} \tilde{V}_{X}} & \sigma_{\tilde{V}_{Z} \tilde{V}_{Y}} & \sigma_{\tilde{V}_{Z}}^{2}
\end{array}\right)=\left(\mathbf{A}^{T} \cdot \mathbf{C}_{\mathbf{e e}}^{-\mathbf{1}} \cdot \mathbf{A}\right)^{-1}
$$

2 The explicit expressions resulting from the matrix operations given in equations (23)

3 and (24) are shown in Appendix B. The variance-covariance matrix is symmetric and

4 positive definite too. Its diagonal elements are positive and have the largest modulus.

5 On the other hand, it is not difficult to prove mathematically (see Appendix C) that the

6 diagonal elements of (24) $\left(\sigma_{\tilde{V}_{X}}^{2}, \sigma_{\tilde{V}_{Y}}^{2}\right.$ and $\left.\sigma_{\tilde{V}_{z}}^{2}\right)$, the variances, are smaller or equal than

7 those of the covariance matrix (18) $\left(\sigma_{\hat{V}_{X}}^{2}, \sigma_{\hat{V}_{Y}}^{2}\right.$ and $\left.\sigma_{\hat{V}_{Z}}^{2}\right)$, that in turn have the largest

8 modulus in that matrix. Hence, in general, it is expected that the WLS solution provide

9 more robust estimates (lower variances and covariances) than the SLS solution.

10 Again the analytical formulae for expressing the weighted least squares solution

11 of (4) are unmanageable and it is preferable to use matrix expressions based on the 12 XYZ equations:

$$
\mathbf{C}_{\tilde{\mathbf{V}} \tilde{\mathbf{V}}_{E N U}}=\mathbf{N} \cdot \mathbf{C}_{\tilde{\mathbf{V}}_{\tilde{X}}{ }_{X Z}} \cdot \mathbf{M}=\mathbf{M}^{T} \cdot \mathbf{C}_{\tilde{\mathbf{V}}_{\tilde{X}} \tilde{X Y Z}} \cdot \mathbf{M}
$$

with $\tilde{\mathbf{V}}_{X Y Z}$ and $\mathbf{C}_{\tilde{\mathbf{V}}_{X Y Z}}$ given by (23) and (24), respectively.

\section{Results: processing an ADCP dataset}

19 Research and Development Institute (SARDI) deployed an upward looking RDI

$20300 \mathrm{kHz}$ WorkHorse (broadband) ADCP off Port Lincoln, South Australia (Figure 2).

21 The mooring was part of the observational work done for the Aquafin CRC: Risk and 
1 Response project. The region offshore of Port Lincoln is used for intensive tuna

2 (southern bluefin tuna, Thunnus maccoyii) ranching activities.

The ADCP was deployed in the Tuna Ranching Zone at a depth of 22m (Figure

2), the primary goal was to estimate wave parameters with the aim of studying the effects of wave induced sediment resuspension. Such events, have in the past, lead to high mortality rates in the farmed tuna. Consequently, it was setup to sample at the fastest rate possible $(2 \mathrm{~Hz})$ in BEAM coordinates. The ADCP recorded data continuously from July $12^{\text {th }}$ at $12: 00: 00$ (UTC) to July $19^{\text {th }}$ at 02:29:08 (UTC) summing up 4x1141107 radial beam velocity profiles. The ADCP was not tripod mounted, rather it was simply attached to a stainless-steel plate, $0.5 \mathrm{~m}^{2}$ and $0.5 \mathrm{~cm}$ thick, ballasted with lead bars. This configuration created a low center of mass and permitted velocity measurements closer to the seafloor. The bin (cell) size specified in the configuration was 1 meter, the blank after transmit distance was $1.74 \mathrm{~m}$ and the center of the first bin was located $3.21 \mathrm{~m}$ above the sea bottom. In order to reduce the rejected raw data, the screening parameters were configured with loose values: correlation threshold 255 counts, RDI error velocity $5 \mathrm{~m} / \mathrm{s}$ and minimum percent good 0 .

The SLS ( $\hat{u}, \hat{v}$ and $\hat{w}$, black lines) and WLS ( $\tilde{u}, \tilde{v}$ and $\tilde{w}$, grey lines) solutions are presented in Figure 3, using ENU coordinates calculated from the 4x1141107 beam radial velocities sensed by the current meter at 12.21m (bin number 9) above the seafloor. The bin level has been chosen arbitrary, there is not much vertical velocity shear neither we have considered any other dynamic constraint for this selection, we have simply taken a middle depth as an example. For this transformation from BEAM to ENU coordinates, the heading, pitch and roll angles time series measured by the internal attitude sensors of the instrument were used. Interestingly, the ADCP remained practically level, both pitch and roll were quite low and stable with mean values of - 
$1 \quad 0.11^{\circ}$ and $-0.63^{\circ}$ and standard deviations of $0.032^{\circ}$ and $0.067^{\circ}$, respectively. The mean

2 heading during the deployment was $200.52^{\circ}$ with a standard deviation of $0.252^{\circ}$. The time series of Figure 3 corresponds to unfiltered data, the whole record has been plotted. In addition to the evident diurnal/semidiurnal tidal modulations in the current, there are spikes of velocity (with values well above $150-200 \mathrm{~cm} \cdot \mathrm{s}^{-1}$ ) distributed along the record, as it was expected from the loose values configured in the screening parameters. Also, it seems that the weighted least squares solutions present fewer and lower spikes, more evident in the energetic phases of the tide. We can focus on the histograms of Figure 4 and on the evolution of the black line. They represent the frequency (number of cases) of velocity classes (every $5 \mathrm{~cm} / \mathrm{s}$ ) for the ENU WLS solutions and their difference with the corresponding ENU SLS solution frequencies, respectively. From the frequency differences, it is observed that the WLS solution has more cases (negative difference) in the lower velocity classes and fewer cases (positive difference) in the higher velocity cases, reducing the tails of the histograms. Although these time series are not normally distributed, we will resort to means and standard deviations as statistics to characterize them. In that regard, the means for each solution method are quite similar among velocity components while the standard deviations are slightly lower using the WLS solution (Table 1).

The advantage of the least squares approximation (section 4) over the standard solution (section 3), is the addition of variance and covariance estimates. On the other hand, we have seen (section 5) that, theoretically, the WLS method should provide lower variance and covariance estimates than the SLS method. In Figure 5 are shown the histograms, in absolute frequencies (number of cases), for the variances and covariances estimated with eq. (26) that corresponds to the ENU WLS solutions of Figure 4 (i.e. to the $4 \times 1141107$ beam radial velocities recorded by the current meter at 
1 12.21m). The frequency difference (difference in the number of cases for each

2 variance/covariance class) with the SLS solutions is also displayed as a black line over

3 the histograms. As it was theoretically predicted, the inspection of this graphics

4 confirms that the variances and covariances produced by the WLS method are generally

5 lower than those calculated with the SLS. The mean variances for the whole time series

6 of WLS solutions and their corresponding standard deviations are approximately a 4\%

7 and a 31\%, respectively, lower than the SLS ones (Table 2). If instead of calculating the

8 means and standard deviations of the variances for the whole time series (8 days) we

9 chose a smaller and more energetic period (from Jul 13 18:00 to Jul 14 06:00, twelve

10 hours) the reduction will rise to a $7 \%$ and a $40 \%$ (data not shown in Table 2),

11 respectively.

\section{A short discussion: WLS solution utility}

The main drawback in the measurements of 3D currents with an ADCP is the adoption of the homogeneous field assumption since it is ineluctable. Thus, the best way to deal with it, is to somehow verify the assumption with the information provided by the ADCP as, for example, Lu and Lueck (1999a) have done. The logical progression in the ADCP data processing, will be the screening of the raw data using parameters such as: echo intensity maximum threshold to implement the "fish rejection", minimum threshold for the percent good, minimum threshold for the correlation and a minimum 21 threshold for the RDI error velocity.

This screening process will certainly increase the quality of the data and should

23 always be done. Another correction techniques, as the bin mapping (Ott, 2002), also

24 should be taken into account. However, when there is an opportunity to estimate an error for each component of the current velocity, it should be calculated and used. The 
1 existence of an error velocity estimate could improve and make much more intuitive for

2 ADCP's end users the identification and rejection of erroneous data. But most

3 importantly, the background reasoning is clear and it is as simple. It is difficult, as it

4 always has been in science, to: provide observations, measures, and estimations and

5 their corresponding measuring errors. The obvious weak point in all this derivation is

6 the quality of the radial beam velocity variances provided by eq. (19) and based on the

7 correlation values recorded by the ADCP. The error estimated by the SLS and the WLS

8 is as good as the quality of the radial beam velocity variances. In that regard, the correct

9 evaluation of eq. (19) is far from the field of knowledge of the authors and, therefore,

10 out of the article scope.

11 As an example of the utility of the least squares proposed solutions, we have

12 screened the time series of the SLS $(\hat{u}, \hat{v}, \hat{w})$ and WLS $(\tilde{u}, \tilde{v}, \tilde{w})$ current velocities

13 introduced in the former section with the following thresholds:

$14 \sigma_{\hat{u}}, \sigma_{\tilde{u}}, \sigma_{\hat{v}}, \sigma_{\tilde{v}}>20 \mathrm{~cm} \cdot \mathrm{s}^{-1}$ and $\sigma_{\hat{w}}, \sigma_{\tilde{w}}>6 \mathrm{~cm} \cdot \mathrm{s}^{-1}$. Every SLS or WLS record with a

15 standard deviation value, of any of the three velocity components, above any of the

16 former thresholds was rejected. After the filter was applied there were 128298 rejections

17 (11.25\%) in the SLS time series and 71351 (6.25\%) in the WLS. By observing the last

18 two rows of Table 1 and Table 2 it is noted that the differences in the means and

19 standard deviations between the unfiltered and filtered WLS solutions are always lower

20 than differences for the SLS filtered and unfiltered solutions. That is, one needs to purge

21 fewer data points using the WLS than with the SLS solutions, 50\% less in this case with

22 the selected thresholds.

23 In addition, to facilitate the data screening process, the estimation of errors

24 should play an important role in the improvement of the methods to analyze, process

25 and map Lowered ADCP (LADCP) (Fischer and Visbeck, 1993; Visbeck, 2002; 
1 Yoshinari et al., 2004) and Vessel Mounted ADCP (Pérez et al., 2003) data. It will also

2 facilitate the estimations of errors for the currents provided by these instruments and,

3 since their output is currently being used in oceanic inverse box models (Joyce et al.,

4 2001; Lherminier et al., 2007), will resort in better oceanic transports and associated

5 errors estimates.

6 Another type of applications that may gain an advantage with the WLS solutions

7 is the measurement of Reynolds stresses with ADCPs and the variance method

8 (Lhermitte and Lemmin, 1994; Lohrmann et al., 1990; Lu and Lueck, 1999b; Rippeth et

9 al., 2002; Stacey et al., 1999; Williams and Simpson, 2004). In order to obtain the

10 Reynolds stresses from ADCP recordings it is necessary to have an estimate of the noise

11 base level (Stacey et al., 1999; Williams and Simpson, 2004). This means that having a

12 more robust way, as the WLS, to estimate velocities and their variances/covariances will

13 greatly improve the quality of the Reynolds stress calculus. But, the exploitation of this

14 idea has enough entity to be the topic of another article and we will not give more

15 details here.

\section{8. Conclusions}

Although the beam to current velocity components standard solution has been, and will keep, working very well for many years we have tried to find an improvement

20 relying on least squares techniques. We have shown that the four beam standard

21 solution, is the same as the least square solution presented in section 4 . While the

22 standard solution gives an indication (the RDI error velocity) of the horizontal

23 homogeneity assumption (and transducer malfunction), the least squares solution

24 provides us with a variance-covariance matrix of the velocity estimates and, at the same 
1 time, does not preclude the calculation of the error velocity for evaluating the quality of

2 the recorded data.

3 Finally a weighted least square solution has been presented. The WLS solution is

4 a more general and robust solution than the standard solution. It is more general

5 because, despite we have presented it with a specific weighting matrix, there is no

6 reason to use other weighting matrix that, for example, takes into account the echo

7 intensity in order to reduce the fish effects. And it is more robust because the weighting

8 matrix is a way of use more information to select the optimum solution and estimate the

9 variance-covariance matrix with smaller variances and covariances than the SLS. This

10 has resulted in the rejection of $50 \%$ less data than using the standard solution. And in an

11 application such as a lowered ADCP would result in a more robust estimate of the 3D

12 current field.

14 Acknowledgements. We thank Arthur D. Johnson Jr. from RDI (Field Service 15 Engineer, Customer Service Group) for his kindly and informative e-mails about the 16 RDI velocity error. We wish to acknowledge the contribution of E. Firing in offering 17 valuable suggestions and stimulating discussions that have helped to improve the 18 manuscript. Support for this work came from the Spanish Science and Technology 19 Ministry (grant MAR1999-1039-W2-01, DYBAGA project), Xunta de Galicia (grant 08MMA014402PR, DIPLOMACIA project) and also the Australian Aquafin CRC:

21 Risk and Response Project. We also wish to thank the staff of the Flinders University

22 School of Chemistry, Physics and Earth Sciences Mechanical Workshop for the help in 23 constructing the moorings, especially Brenton Perkins and Bob Northeast. To the 24 skipper of the RV Breakwater Bay, Brenton Ebert. And also Tyson Gilbert and Kate 25 Rodda for their help in the retrieval of the mooring. 


\section{Appendix A}

The transformation matrix needed to convert the velocities from the instrument

4 coordinate system (XYZ, $\overrightarrow{\mathbf{V}}_{X Y Z}=\left\{V_{X}, V_{Y}, V_{Z}\right\}$ ) to the East/North/Up system (ENU)

5 relays on the tilt angles between the XYZ axis and the ENU ones $\left(\overrightarrow{\mathbf{V}}_{E N U}=\{u, v, w\}\right)$. For

6 an upward looking RDI ADCP this matrix $\mathbf{N}$ is (R. D. Instruments, 1997):

7

8

$$
\overrightarrow{\mathbf{V}}_{E N U}=\mathbf{N} \cdot \overrightarrow{\mathbf{V}}_{X Y Z}
$$

$9 \quad \mathbf{N}=\left(\begin{array}{ccc}-\cos \theta \cdot \cos \varphi-\sin \theta \cdot \sin \phi \cdot \sin \varphi & \sin \theta \cdot \cos \phi & \sin \theta \cdot \sin \phi \cdot \cos \varphi-\cos \theta \cdot \sin \varphi \\ \sin \theta \cdot \cos \varphi-\cos \theta \cdot \sin \phi \cdot \sin \varphi & \cos \theta \cdot \cos \phi & \cos \theta \cdot \sin \phi \cdot \cos \varphi+\sin \theta \cdot \sin \varphi \\ \cos \phi \cdot \sin \varphi & \sin \phi & -\cos \phi \cdot \cos \varphi\end{array}\right)$

11 where $\theta$ is the heading (rotational angle about Z-axis) given by the ADCP compass, $\varphi$ is

12 the roll (rotational angle about Y-axis) recorded by the ADCP-roll internal sensor, $\phi$ is

13 the true pitch (rotational angle about X-axis) calculated with the ADCP-pitch internal

14 sensor $\psi$ and the roll through the expression $\phi=\arctan (\tan \psi \cdot \cos \varphi)$. The inverse

15 transformation, from ENU velocities to XYZ velocities, is given by $\mathbf{M}$ the inverse of $\mathbf{N}$ :

$$
\overrightarrow{\mathbf{V}}_{X Y Z}=\mathbf{N}^{-1} \cdot \overrightarrow{\mathbf{V}}_{E N U}=\mathbf{M} \cdot \overrightarrow{\mathbf{V}}_{E N U}
$$

$17 \quad \mathbf{M}=\left(\begin{array}{ccc}-\cos \theta \cdot \cos \varphi-\sin \theta \cdot \sin \phi \cdot \sin \varphi & \sin \theta \cdot \cos \varphi-\cos \theta \cdot \sin \phi \cdot \sin \varphi & \cos \phi \cdot \sin \varphi \\ \sin \theta \cdot \cos \phi & \cos \theta \cdot \cos \phi & \sin \phi \\ \sin \theta \cdot \sin \phi \cdot \cos \varphi-\cos \theta \cdot \sin \varphi & \cos \theta \cdot \sin \phi \cdot \cos \varphi+\sin \theta \cdot \sin \varphi & -\cos \phi \cdot \cos \varphi\end{array}\right)$ 


\section{Appendix B}

$$
\begin{aligned}
& \left(\mathbf{A}^{T} \cdot \mathbf{C}_{\mathrm{ee}}^{-1} \cdot \mathbf{A}\right)^{-1} \cdot \mathbf{A}^{T} \cdot \mathbf{C}_{\mathrm{ee}}^{-1} \cdot \overrightarrow{\mathbf{b}}= \\
& =\left(\begin{array}{c}
\frac{\left(2 \cdot \sigma_{b_{2}}^{2}+\sigma_{b_{3}}^{2}+\sigma_{b_{4}}^{2}\right) \cdot b_{1}-\left(2 \cdot \sigma_{b_{1}}^{2}+\sigma_{b_{3}}^{2}+\sigma_{b_{4}}^{2}\right) \cdot b_{2}+\left(\sigma_{b_{1}}^{2}-\sigma_{b_{2}}^{2}\right) \cdot b_{3}+\left(\sigma_{b_{1}}^{2}-\sigma_{b_{2}}^{2}\right) \cdot b_{4}}{2 \cdot \sin \beta \cdot \sum_{i=1}^{4} \sigma_{b_{i}}^{2}} \\
\frac{\left(\sigma_{b_{4}}^{2}-\sigma_{b_{3}}^{2}\right) \cdot b_{1}+\left(\sigma_{b_{4}}^{2}-\sigma_{b_{3}}^{2}\right) \cdot b_{2}-\left(\sigma_{b_{1}}^{2}+\sigma_{b_{2}}^{2}+2 \cdot \sigma_{b_{4}}^{2}\right) \cdot b_{3}+\left(\sigma_{b_{1}}^{2}+\sigma_{b_{2}}^{2}+2 \cdot \sigma_{b_{3}}^{2}\right) \cdot b_{4}}{2 \cdot \sin \beta \cdot \sum_{i=1}^{4} \sigma_{b_{i}}^{2}} \\
\frac{\left(\sigma_{b_{3}}^{2}+\sigma_{b_{4}}^{2}\right) \cdot b_{1}+\left(\sigma_{b_{3}}^{2}+\sigma_{b_{4}}^{2}\right) \cdot b_{2}+\left(\sigma_{b_{1}}^{2}+\sigma_{b_{2}}^{2}\right) \cdot b_{3}+\left(\sigma_{b_{1}}^{2}+\sigma_{b_{2}}^{2}\right) \cdot b_{4}}{2 \cdot \cos \beta \cdot \sum_{i=1}^{4} \sigma_{b_{i}}^{2}}
\end{array}\right)
\end{aligned}
$$

$$
\left(\mathbf{A}^{T} \cdot \mathbf{C}_{\mathbf{e e}}^{-1} \cdot \mathbf{A}\right)^{-1}=
$$

$$
\frac{4 \cdot \sigma_{b_{1}}^{2} \cdot \sigma_{b_{2}}^{2}+\left(\sigma_{b_{1}}^{2}+\sigma_{b_{2}}^{2}\right) \cdot\left(\sigma_{b_{3}}^{2}+\sigma_{b_{4}}^{2}\right)}{\sin ^{2} \beta \cdot 4 \cdot \sum_{i=1}^{4} \sigma_{b_{i}}^{2}}
$$

$$
\frac{\left(\sigma_{b_{2}}^{2}-\sigma_{b_{1}}^{2}\right) \cdot\left(\sigma_{b_{3}}^{2}-\sigma_{b_{4}}^{2}\right)}{\sin ^{2} \beta \cdot 4 \cdot \sum_{i=1}^{4} \sigma_{b_{i}}^{2}}
$$

$\frac{\left(\sigma_{b_{1}}^{2}-\sigma_{b_{2}}^{2}\right) \cdot\left(\sigma_{b_{3}}^{2}+\sigma_{b_{4}}^{2}\right)}{\left(\sigma^{4}\right.}$

$$
\frac{\left(\sigma_{b_{2}}^{2}-\sigma_{b_{1}}^{2}\right) \cdot\left(\sigma_{b_{3}}^{2}-\sigma_{b_{4}}^{2}\right)}{\sin ^{2} \beta \cdot 4 \cdot \sum_{i=1}^{4} \sigma_{b_{i}}^{2}}
$$

$\sin \beta \cdot \cos \beta \cdot 4 \cdot \sum_{i=1}^{4} \sigma_{b_{i}}^{2}$

$$
\frac{\left(\sigma_{b_{1}}^{2}-\sigma_{b_{2}}^{2}\right) \cdot\left(\sigma_{b_{3}}^{2}+\sigma_{b_{4}}^{2}\right)}{\sin \beta \cdot \cos \beta \cdot 4 \cdot \sum_{i=1}^{4} \sigma_{b_{i}}^{2}}
$$

$$
\left.\begin{array}{cc}
\frac{4 \cdot \sigma_{b_{3}}^{2} \cdot \sigma_{b_{4}}^{2}+\left(\sigma_{b_{1}}^{2}+\sigma_{b_{2}}^{2}\right) \cdot\left(\sigma_{b_{3}}^{2}+\sigma_{b_{4}}^{2}\right)}{\sin ^{2} \beta \cdot 4 \cdot \sum_{i=1}^{4} \sigma_{b_{i}}^{2}} & \frac{\left(\sigma_{b_{1}}^{2}+\sigma_{b_{2}}^{2}\right) \cdot\left(\sigma_{b_{4}}^{2}-\sigma_{b_{3}}^{2}\right)}{\sin \beta \cdot \cos \beta \cdot 4 \cdot \sum_{i=1}^{4} \sigma_{b_{i}}^{2}} \\
\frac{\left(\sigma_{b_{1}}^{2}+\sigma_{b_{2}}^{2}\right) \cdot\left(\sigma_{b_{4}}^{2}-\sigma_{b_{3}}^{2}\right)}{\sin \beta \cdot \cos \beta \cdot 4 \cdot \sum_{i=1}^{4} \sigma_{b_{i}}^{2}} & \frac{\left(\sigma_{b_{1}}^{2}+\sigma_{b_{2}}^{2}\right) \cdot\left(\sigma_{b_{3}}^{2}+\sigma_{b_{4}}^{2}\right)}{\cos ^{2} \beta \cdot 4 \cdot \sum_{i=1}^{4} \sigma_{b_{i}}^{2}}
\end{array}\right)
$$




\section{Appendix C}

2

One way to proof that the WLS variances are always lower or equal to those

3 obtained with the SLS solution is simply to take their corresponding expressions from

4 equations (24) and (18), respectively, and show that their differences (SLS minus WLS)

5 are always positive or null:

6

$$
\sigma_{\hat{V}_{X}}^{2}-\sigma_{\tilde{V}_{X}}^{2}=\frac{\left(\sigma_{b_{1}}-\sigma_{b_{2}}\right)^{2}}{\sin ^{2} \beta \cdot 4 \cdot \sum_{i=1}^{4} \sigma_{b_{i}}^{2}} \geq 0
$$

7

8

$$
\sigma_{\hat{V}_{Y}}^{2}-\sigma_{\tilde{V}_{Y}}^{2}=\frac{\left(\sigma_{b_{3}}-\sigma_{b_{4}}\right)^{2}}{\sin ^{2} \beta \cdot 4 \cdot \sum_{i=1}^{4} \sigma_{b_{i}}^{2}} \geq 0
$$

9

10

$$
\sigma_{\hat{V}_{Z}}^{2}-\sigma_{\tilde{V}_{Z}}^{2}=\frac{\left[\left(\sigma_{b_{3}}+\sigma_{b_{4}}\right)-\left(\sigma_{b_{1}}+\sigma_{b_{2}}\right)\right]^{2}}{16 \cdot \cos ^{2} \beta \cdot \sum_{i=1}^{4} \sigma_{b_{i}}^{2}} \geq 0
$$




\section{References}

2

3

4

5

6

7

8

9

10

11

12

13

14

15

16

17

18

19

20

21

22

23

24

25

26

27

28

29

30

Bevington, P.R., Robinson, D.K., 1992. Data reduction and error analysis for the physical scences. McGraw-Hill, New York.

Brumley, B.H., Cabrera, R.G., Deines, K.L., Terray, E.A., 1991. Performance of a Broad-Band Acoustic Doppler Current Profiler. IEEE Journal of Oceanic Engineering 16 (4), 402-407.

Chereskin, T.K., Harding, A.J., 1993. Modeling the performance of an Acoustic Doppler Current Profiler. Journal of Atmospheric and Oceanic Technology 10 (1), 4163.

Fischer, J., Visbeck, M., 1993. Deep velocity profiling with self-contained ADCPs. Journal of Atmospheric and Oceanic Technology 10, 764-773.

Gargett, A.E., 1994. Observing turbulence with a modified Acoustic Doppler Current Profiler. Journal of Atmospheric and Oceanic Technology 11 (6), 1592-1610.

Gordon, R.L., 1996. Acoustic Doppler Current Profiler. Principles of operation. A practical primer. RD Instruments, San Diego, p. 57.

Hamilton, J.M., Fowler, G.A., Belliveau, D.J., 1997. Mooring vibration as a source of current meter error and its correction. Journal of Atmospheric and Oceanic Technology 14 (3 pt 2), 644-655.

Hummon, J.M., Firing, E., 2003. A direct comparison of two RDI shipboard ADCPs: a $75-\mathrm{kHz}$ Ocean Surveyor and a 150-kHz Narrow Band. Journal of Atmospheric and Oceanic Technology 20 (6), 872-888.

Hurther, D., Lemmin, U., 2001. A correction method for turbulence measurements with a 3D Acoustic Doppler Velocity Profiler. Journal of Atmospheric and Oceanic Technology 18 (3), 446-458.

Joyce, T.M., Hernandez-Guerra, A., Smethie Jr., W.M., 2001. Zonal circualtion in NW Atlantic and Caribbean from a meridional World Ocean Circulation Experiment hydrographic section at $66^{\circ} \mathrm{W}$. Journal of Geophysical Research 106 (C10), 2209522113.

Lherminier, P., Mercier, H., Gourcuff, C., Alvarez, M., Bacon, S., Kermabon, C., 2007. Transports across the 2002 Greenland-Portugal Ovide section and comparison with 1997. Journal of Geophysical Research 112 (C07), 20.

Lhermitte, R., Lemmin, U., 1994. Open-channel flow and turbulence measurement by high-resolution Doppler sonar. Journal of Atmospheric and Oceanic Technology 11 (5), 1295-1308. 
Lhermitte, R., Serafin, R., 1984. Pulse-to-pulse coherent doppler sonar signal processing techniques. Journal of Atmospheric and Oceanic Technology 1 (4), 293-308.

Lohrmann, A., Hackett, B., Røed, L.P., 1990. High resolution measurements of turbulence, velocity and stress using a pulse-to-pulse coherent sonar. Journal of Atmospheric and Oceanic Technology 7 (1), 19-37.

Lu, Y., Lueck, R.G., 1999a. Using a broadband ADCP in a tidal channel. Part I: Mean flow and shear. Journal of Atmospheric and Oceanic Technology 16 (11), 1556-1567.

Lu, Y., Lueck, R.G., 1999b. Using a broadband ADCP in a tidal channel. Part II: Turbulence. Journal of Atmospheric and Oceanic Technology 16 (11), 1568-1579.

New, A.L., 1992. Factors affecting the quality of shipboard acoustic Doppler current profiler data. Deep-Sea Research 39 (11/12), 1985-1996.

Ott, M.W., 2002. An improvement in the calculation of ADCP velocities. Journal of Atmospheric and Oceanic Technology 19 (10), 1738-1741.

Ott, M.W., 2005. The accuracy of acoustic vertical velocity measurements: instrument biases and the effect of zooplankton migration. Continental Shelf Research 25 (2), 243257.

Ott, M.W., Dewey, R., Garrett, C., 2002. Reynold stresses and secondary circulation in a stratified rotating shear flow. journal of Physical Oceanography 32 (11), 3249-3268.

Pérez, F.F., Gilcoto, M., Ríos, A.F., 2003. Large and mesoscale variability of the water masses and the deep chlorophyll maximum in the Azores Front. Journal of Geophysical Research 108 (C7), 3215-3233.

Pinkel, R., Smith, J.A., 1992. Repeat-sequence coding for improved precision of Doppler Sonar and Sodar. Journal of Atmospheric and Oceanic Technology 9 (2), 149163.

R. D. Instruments, 1996. Broadband ADCP advanced principles of operation. RD Instruments, San Diego, p. 15.

R. D. Instruments, 1997. ADCP coordinate transformation. Formulas and calculations. RD Instruments, San Diego, p. 26.

Rippeth, T.P., Williams, E., Simpson, J.H., 2002. Reynolds stress and turbulent energy production in a tidal channel. Journal of Physical Oceanography 32 (4), 1242-1251.

Stacey, M.T., Monismith, S.G., Burau, J.R., 1999. Observations of turbulence in a partially stratified estuary. Journal of Physical Oceanography 29 (8), 1950-1970.

van Haren, H., 2001. Estimates of sea level, waves and winds from a bottom-mounted ADCP in a shelf sea. Journal of Sea Research 45 (1), 1-14. 
1 Visbeck, M., 2002. Deep velocity profiling using Lowered Acoustic Doppler Current

2 Profilers: bottom track and inverse solutions. Journal of Atmospheric and Oceanic

3 Technology 19 (5), 794-807.

4

5 Visbeck, M., Fischer, J., 1995. Sea surface conditions remotely sensed by upward6 looking ADCPs. Journal of Atmospheric and Oceanic Technology 12 (1), 141-149.

7

Williams, E., Simpson, J.H., 2004. Uncertainties in estimates of Reynolds stress and TKE production rate using the ADCP Variance Method. Journal of Atmospheric and 10 Oceanic Technology 21 (2), 347-357.

11

12 Wunsch, C., 1996. The ocean circulation inverse problem. Cambridge University Press, 13 Cambridge.

14

Yoshinari, H., Yasuda, I., Ikeda, M., 2004. Meridional transport of North Pacific 16 Intermediate Water across $37^{\circ} \mathrm{N}$ based on an objective analysis of lowered acoustic 17 Doppler current profiler data. Journal of Geophysical Research 109 (C02023), 16. 
Figure 1 Side (left) and plan (right) views of a four-beam ADCP. The transducer ceramics are labeled from T1 to T4. The instrument XYZ coordinate system is drawn over the ADCP head in both views. Two actual current velocities $\overrightarrow{\mathbf{V}}_{4}$ and $\overrightarrow{\mathbf{V}}_{3}$ to be observed by T4 and T3 instrument beams, respectively, at a certain level above the bottom will be sensed as radial beam velocities (projections of the current velocities along the corresponding beam) $\overrightarrow{\mathbf{b}}_{4}$ and $\overrightarrow{\mathbf{b}}_{3}$, respectively. Also, $\overrightarrow{\mathbf{V}}_{4}$ and $\overrightarrow{\mathbf{V}}_{3}$ are decomposed in the corresponding XYZ components according to the transducers' slant angle $\beta$.

Figure 2 Map of the South of Spencer Gulf, South Australia, where Port Lincoln is located (South West). The RDI 300kHz Workhorse ADCP was deployed in the Port Lincoln tuna farm region, Northeastward of Boston I. (open diamond).

Figure 3 Time series of the SLS (black lines) and WLS (grey lines) ENU estimations of the East (top), North (middle) and vertical (bottom) components of the currents obtained from the radial beam velocities measured with the ADCP deployment off Port Lincoln.

Figure 4 Velocity histograms, in absolute frequencies (left Y-axis, number of cases), of the ENU-WLS North (top), East (middle) and vertical (bottom) velocity component estimations (eq. (25)) generated from the 1141107 four-beam ADCP measurements. The velocity classes were distributed every $5 \mathrm{~cm} / \mathrm{s}$. In every plot, the black line shows the frequency difference (right Y-axis, in number of cases) for each velocity class between the ENU-WLS histogram and the corresponding ENU-SLS histogram (not shown, velocity estimations made with (eq. (21))).

Figure 5 Histograms, in absolute frequencies (left Y-axis, number of cases), of the ENU-WLS variances (plots a) $\sigma_{\tilde{u}}^{2}$, b) $\sigma_{\tilde{v}}^{2}$,and c) $\sigma_{\tilde{w}}^{2}$ ) and covariances (plots d) $\sigma_{\tilde{u} \tilde{v}}=\sigma_{\tilde{v} \tilde{u}}$, e) $\sigma_{\tilde{u} \tilde{w}}=\sigma_{\tilde{w} \tilde{u}}$, and f) $\sigma_{\tilde{v} \tilde{w}}=\sigma_{\tilde{w} \tilde{v}}$ ) estimations (eq. (26)) generated from the 1141107 four-beam ADCP measurements. The variance and covariance classes were distributed every $20 \mathrm{~cm}^{2} \cdot \mathrm{s}^{-2}$. In every plot, the black line shows the frequency difference (right Y-axis, in number of cases) for each variance/covariance class between the ENUWLS histogram and the corresponding ENU-SLS histogram (not shown, variance/covariance estimations made with (eq. (22))). 
Table 1 Mean and standard deviations of the unfiltered (firs two rows) and filtered (third and fourth rows) WLS ( $\tilde{u}, \tilde{v}$ and $\tilde{w})$ and SLS ( $\hat{u}, \hat{v}$ and $\hat{w}$ ) velocity estimations. The last two rows are the differences between unfiltered and filtered values.

\begin{tabular}{|l|r|r|r|r|r|r|}
\hline & \multicolumn{1}{|c|}{$\begin{array}{c}\hat{u} \\
\left(\mathrm{~cm} \cdot \mathrm{s}^{-1}\right)\end{array}$} & $\begin{array}{c}\tilde{u} \\
\left(\mathrm{~cm} \cdot \mathrm{s}^{-1}\right)\end{array}$ & $\begin{array}{c}\hat{v} \\
\left(\mathrm{~cm} \cdot \mathrm{s}^{-1}\right)\end{array}$ & $\begin{array}{c}\tilde{v} \\
\left(\mathrm{~cm} \cdot \mathrm{s}^{-1}\right)\end{array}$ & $\begin{array}{c}\hat{w} \\
\left(\mathrm{~cm} \cdot \mathrm{s}^{-1}\right)\end{array}$ & $\begin{array}{c}\tilde{w} \\
\left(\mathrm{~cm} \cdot \mathrm{s}^{-1}\right)\end{array}$ \\
\hline Mean & -0.116 & -0.116 & 0.596 & 0.595 & -0.174 & -0.175 \\
\hline STD & 17.365 & 17.076 & 18.760 & 18.506 & 4.634 & 4.567 \\
\hline Filt. Mean & -0.247 & -0.193 & 0.740 & 0.678 & -0.151 & -0.161 \\
\hline Filt. STD & 16.477 & 16.675 & 17.951 & 18.120 & 4.408 & 4.462 \\
\hline Mean-Filt. Mean & 0.131 & 0.077 & -0.144 & -0.083 & -0.022 & -0.014 \\
\hline STD-Filt. STD & 0.888 & 0.400 & 0.809 & 0.386 & 0.225 & 0.105 \\
\hline
\end{tabular}


Table 2 Mean and standard deviations of the unfiltered (firs two rows) and filtered (third and fourth rows) WLS ( $\sigma_{\tilde{u}}, \sigma_{\tilde{v}}$ and $\left.\sigma_{\tilde{w}}\right)$ and SLS $\left(\sigma_{\hat{u}}, \sigma_{\hat{v}}\right.$ and $\left.\sigma_{\hat{w}}\right)$ velocity standard deviations estimations. The last two rows are the differences between unfiltered and filtered values.

\begin{tabular}{|l|r|r|r|r|r|r|}
\hline & $\begin{array}{c}\sigma_{\hat{u}} \\
\left(\mathrm{~cm} \cdot \mathrm{s}^{-1}\right)\end{array}$ & $\begin{array}{c}\sigma_{\tilde{u}} \\
\left(\mathrm{~cm} \cdot \mathrm{s}^{-1}\right)\end{array}$ & $\begin{array}{c}\sigma_{\hat{v}} \\
\left(\mathrm{~cm} \cdot \mathrm{s}^{-1}\right)\end{array}$ & $\begin{array}{c}\sigma_{\tilde{v}} \\
\left(\mathrm{~cm} \cdot \mathrm{s}^{-1}\right)\end{array}$ & $\begin{array}{c}\sigma_{\hat{w}} \\
\left(\mathrm{~cm} \cdot \mathrm{s}^{-1}\right)\end{array}$ & $\begin{array}{c}\sigma_{\tilde{w}} \\
\left(\mathrm{~cm} \cdot \mathrm{s}^{-1}\right)\end{array}$ \\
\hline Mean & 15.530 & 14.929 & 15.510 & 14.921 & 4.019 & 3.860 \\
\hline STD & 3.720 & 2.477 & 3.541 & 2.446 & 0.837 & 0.556 \\
\hline Filtered Mean & 14.824 & 14.603 & 14.822 & 14.603 & 3.830 & 3.786 \\
\hline Filtered STD & 1.916 & 1.920 & 1.911 & 1.913 & 0.398 & 0.435 \\
\hline Mean-Filt. Mean & 0.706 & 0.326 & 0.688 & 0.318 & 0.190 & 0.074 \\
\hline STD-Filt. STD & 1.804 & 0.558 & 1.630 & 0.533 & 0.439 & 0.121 \\
\hline
\end{tabular}




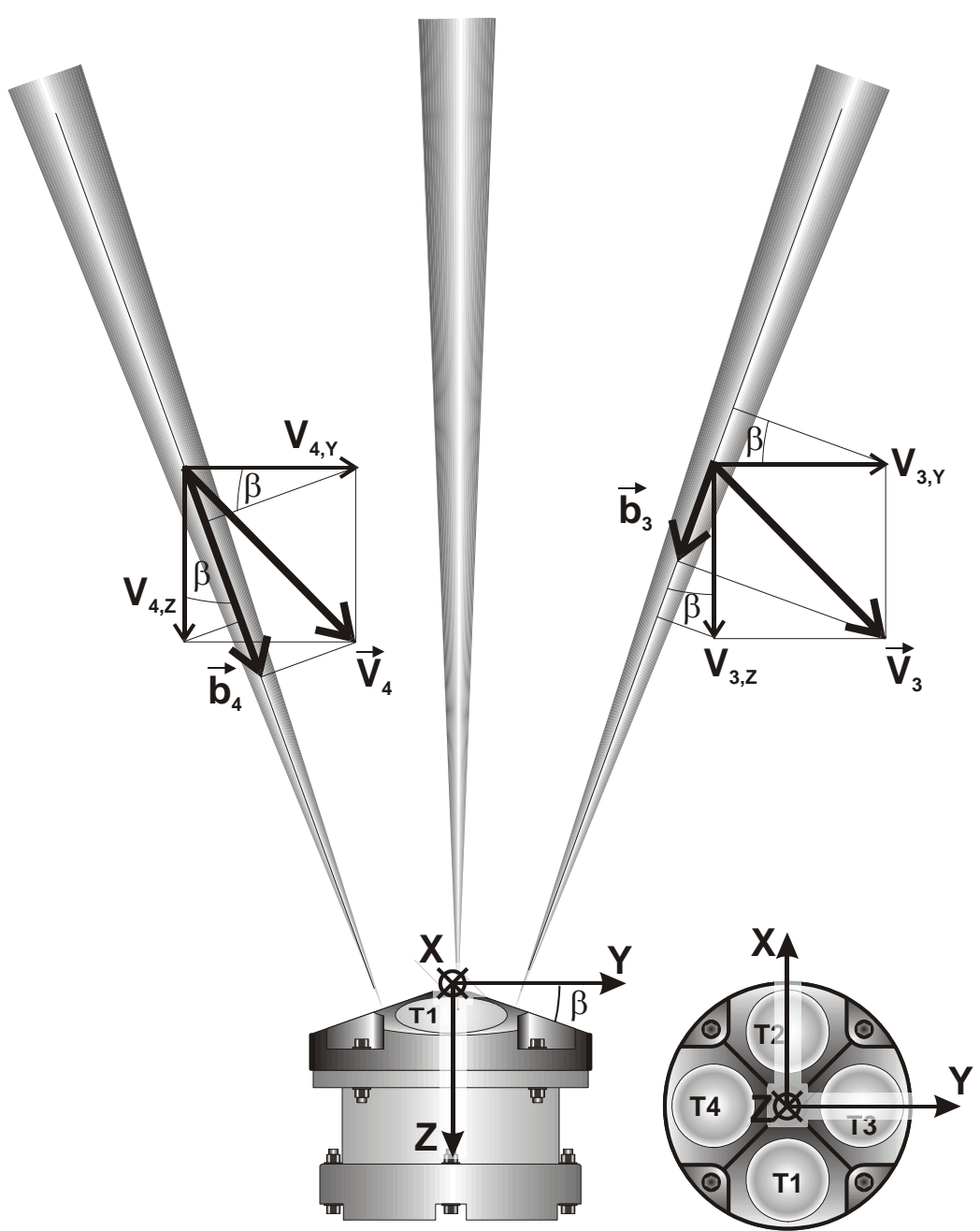

Figure 1 Side (left) and plan (right) views of a four-beam ADCP. The transducer ceramics are labeled from T1 to T4. The instrument XYZ coordinate system is drawn over the ADCP head in both views. Two actual current velocities $\overrightarrow{\mathbf{V}}_{4}$ and $\overrightarrow{\mathbf{V}}_{3}$ to be observed by T4 and T3 instrument beams, respectively, at a certain level above the bottom will be sensed as radial beam velocities (projections of the current velocities along the corresponding beam) $\overrightarrow{\mathbf{b}}_{4}$ and $\overrightarrow{\mathbf{b}}_{3}$, respectively. Also, $\overrightarrow{\mathbf{V}}_{4}$ and $\overrightarrow{\mathbf{V}}_{3}$ are decomposed in the corresponding XYZ components according to the transducers' slant angle $\beta$. 


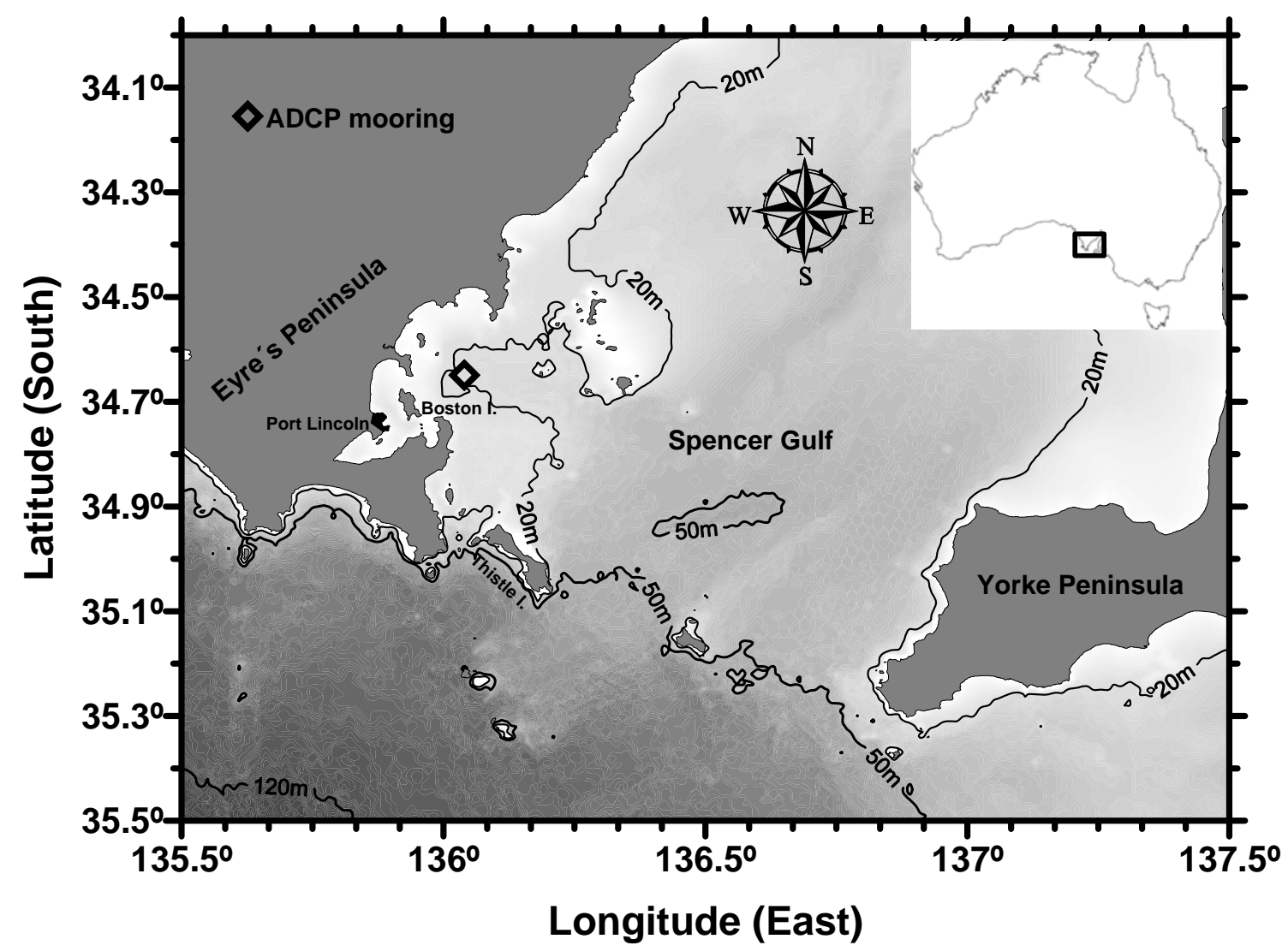

Figure 2 Map of the South of Spencer Gulf, South Australia, where Port Lincoln is located (South West). The RDI 300kHz Workhorse ADCP was deployed in the Port Lincoln tuna farm region, Northeastward of Boston I. (open diamond). 

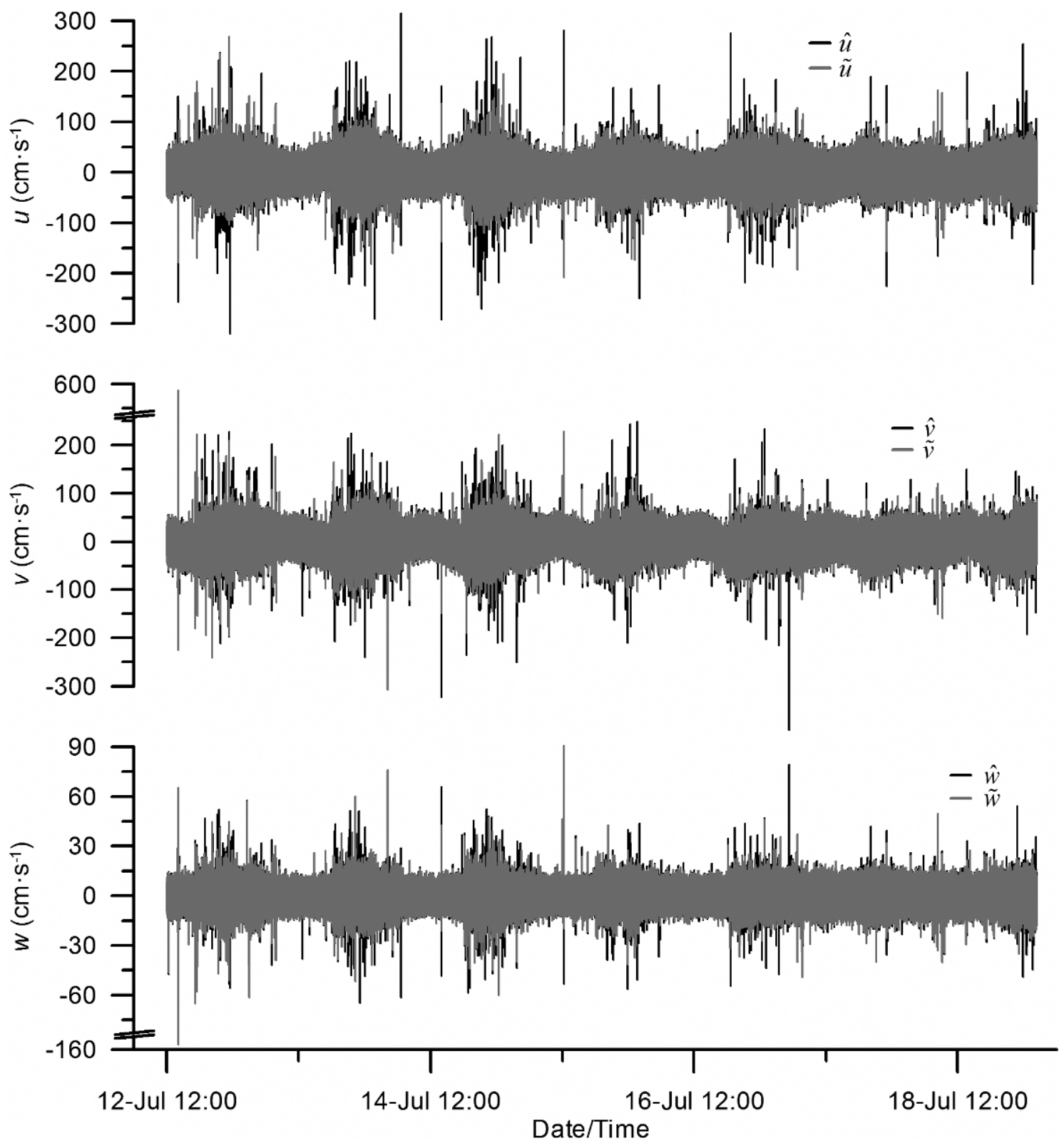

Figure 3 Time series of the SLS (black lines) and WLS (grey lines) ENU estimations of the East (top), North (middle) and vertical (bottom) components of the currents obtained from the radial beam velocities measured with the ADCP deployment off Port Lincoln. 

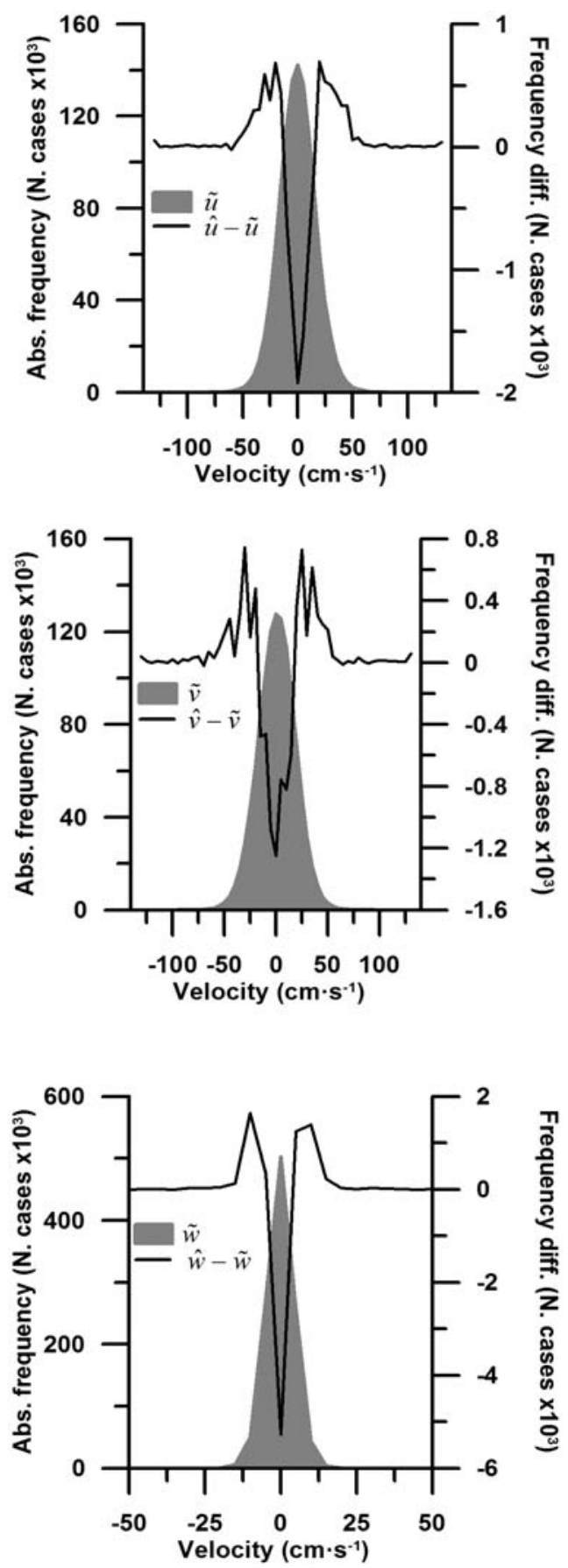

Figure 4 Velocity histograms, in absolute frequencies (left Y-axis, number of cases), of the ENU-WLS North (top), East (middle) and vertical (bottom) velocity component estimations (eq. (25)) generated from the 1141107 four-beam ADCP measurements. The velocity classes were distributed every $5 \mathrm{~cm} / \mathrm{s}$. In every plot, the black line shows the frequency difference (right Y-axis, in number of cases) for each velocity class between the ENU-WLS histogram and the corresponding ENU-SLS histogram (not shown, velocity estimations made with (eq. (21))). 

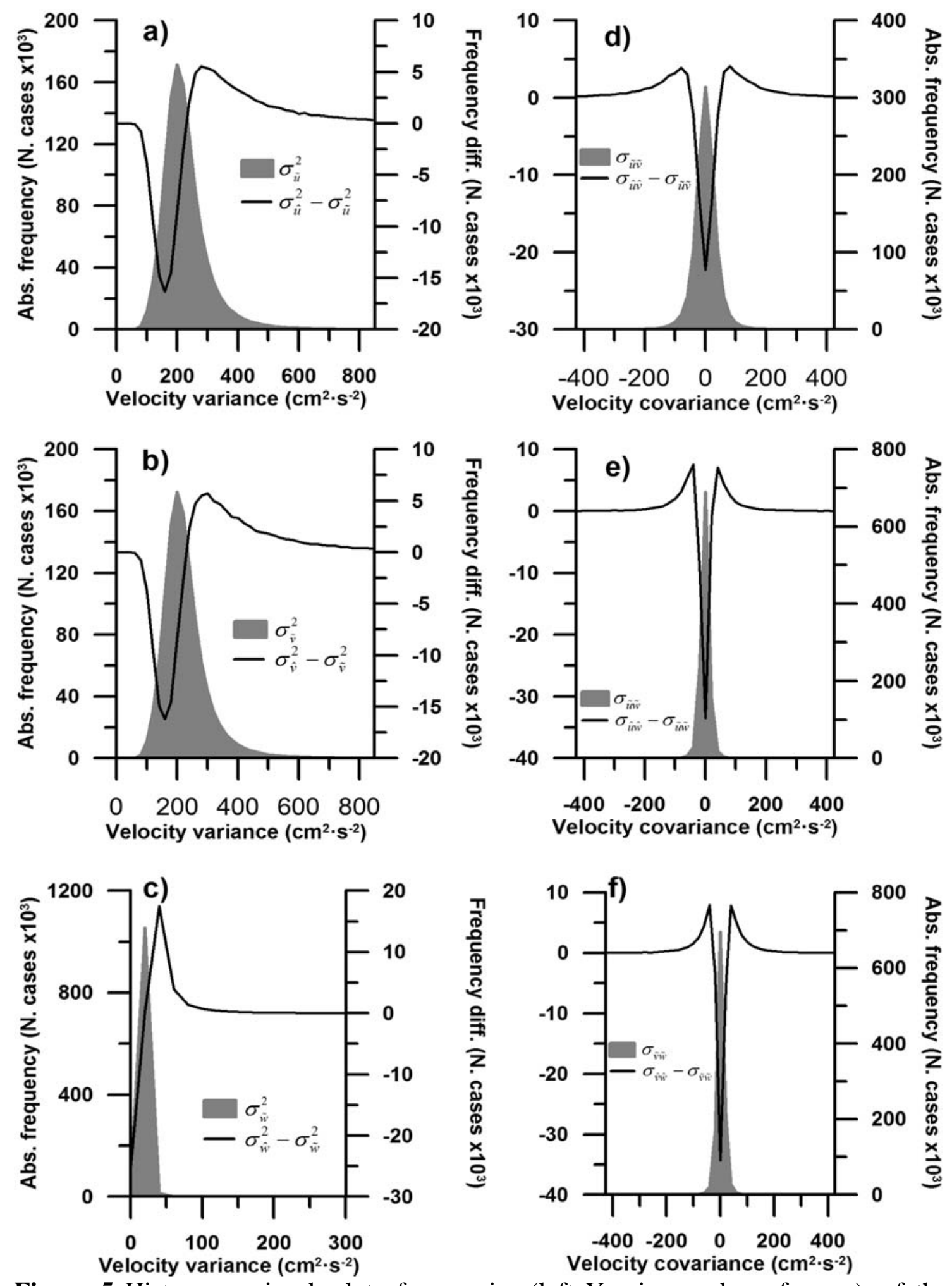

Figure 5 Histograms, in absolute frequencies (left Y-axis, number of cases), of the ENU-WLS variances (plots a) $\sigma_{\tilde{u}}^{2}$, b) $\sigma_{\tilde{v}}^{2}$,and c) $\sigma_{\tilde{w}}^{2}$ ) and covariances (plots d) $\sigma_{\tilde{u} \tilde{v}}=\sigma_{\tilde{v} \tilde{u}}$, e) $\sigma_{\tilde{u} \tilde{w}}=\sigma_{\tilde{w} \tilde{u}}$, and f) $\sigma_{\tilde{v} \tilde{w}}=\sigma_{\tilde{w} \tilde{v}}$ ) estimations (eq. (26)) generated from the 1141107 four-beam ADCP measurements. The variance and covariance classes were distributed every $20 \mathrm{~cm}^{2} \cdot \mathrm{s}^{-2}$. In every plot, the black line shows the frequency difference (right Y-axis, in number of cases) for each variance/covariance class between the ENUWLS histogram and the corresponding ENU-SLS histogram (not shown, variance/covariance estimations made with (eq. (22))). 